\section{Effect of retinoic acid on the nitrergic innervation of meibomian glands in rats}

\author{
A. Bolekova, D. Kluchova, L. Tomasova, \\ N. Hvizdosova \\ Department of Anatomy, Faculty \\ of Medicine, Pavol Jozef Safarik \\ University, Kosice, Slovak Republic
}

\section{Abstract}

The purpose of this study was to investigate the effect of prenatal administration of retinoic acid (RA) on the development of nicotinamide adenine dinucleotide diaphorase (NADPH-d) positive structures in the rat Meibomian glands. One $\mathrm{mg} / \mathrm{kg}$ of RA was applied to pregnant Wistar rats intraperitonaelly during the gestational period in each of the $12^{\text {th }}-14^{\text {th }}$ embryonic days (totally $3 \mathrm{mg} / \mathrm{kg}$ ). Sections of the central upper eyelids were investigated in rat pups on the $14^{\text {th }}$ postnatal day. They were processed histochemically for NADPH-d, to study the presence and distribution of nitric oxide synthase (NOS) positive nerve structures. NADPH-d staining of Meibomian glands was compared in two groups of rat pups. In the control group, eyelids of 14 day-old rats were studied with no experimental intervention. The second group consisted of rat pups which were prenatally administered the excess of RA. Histochemical analysis of control eyelids revealed numerous NADPH-d wellstained acini of Meibomian glands arranged tightly into groups. Intensively stained vessels and NADPH-d/NOS-positive nerve fibers bordered acini of Meibomian glands. These structures were present in the submucosal layer as well. The analysis of RA group showed less numerous, shrunken acini of Meibomian glands that were seen not only smaller in size, but also in density of their staining and the amount of nitrergic nerve fibers around acini were considerably lowered. In the submucosa differences were noticed compared to the control group, there were numerous NADPH-d stained vessels accompanied by NADPH-d/NOSpositive nerve fibers. The excess of RA during the prenatal period may influence on the development and morphology of NADPH-d positive structures of rat's Meibomian glands.

\section{Introduction}

Meibomian glands are large sebaceous glands of the eyelids whose lipid secretions are essential for the maintenance, health and integrity of ocular surface. They have distinct neural innervation and regulation via hormones and other factors. Several studies have reported on the neurotransmitters of the Meibomian glands in different species; in guinea pigs: neuropeptide $\mathrm{Y}$, substance $\mathrm{P}$ and vasoactive intestinal polypeptide (VIP); in humans: calcitonin gene-related peptide (CGRP) and substance P; in cynomolgus monkeys: substance P, CRGP, neuropeptide $\mathrm{Y}$ and VIP; in rat: nitric oxide, enkephalin, tyrosine hydrolase and dopamine-beta-hydroxylase. ${ }^{1,2}$

Meibomian gland dysfunction is common, but yet often overlooked lid margin disease, as a result of altered or reduced lipid excretion that lead to disruption of the tear film with resultant irritation to the cornea, conjunctiva, and lids. ${ }^{3-5}$ It may thus be acknowledged that its importance has conceivably been underestimated, and possibly the most frequently cause of dry eye syndrome could be due to increased evaporation of the aqueous tears. Dry eye syndrome affects about $75 \%$ of population that may not blink so often (work with PC, watching TV or reading) or wear contact lens. ${ }^{1}$ This can also result from chemical burns, different autoimmune diseases, bacteria that may breakdown the oil, or taking oral isotretinoin medications. ${ }^{6,7}$

Toxicity and subtoxicity of vitamins has been associated with overdosing of vitamin supplements, which are added to a variety of food in industrialize countries, or with extremely high doses of multivitamins taken by pregnant women. ${ }^{8}$ Vitamin A and its biologically active metabolite, retinoic acid (RA) are essential for embryonic development, regulate vital functions of the embryo, modulate neurogenesis, neuronal survival and synaptic plasticity. ${ }^{9-11}$ It is very important to have the right balance of vitamin $\mathrm{A}$ in the maternal diet for a successful developmental outcome, because it can act as a teratogen in critical developmental periods. ${ }^{12,13}$ Excess intake of RA shows remarkable teratogenic similarities to those generated by a lack of RA during prenatal periods. ${ }^{12,14}$ As retinoids play a key role in fin formation, differentiation and maturation of many types of cells, they help reverse the pathogenesis of malignancies. ${ }^{6}, 15$ RA promotes cellular differentiation and can induce regeneration in a number of different organ systems therefore it is used for treatment of skin diseases, cancers, immunotherapy and vaccine strategies. ${ }^{16}$ Unfortunately, malformations and set of behavioural anomalies were seen in the human embryo exposed to 13-cis RA during treatment of the mother with the acne drug isotretinoin (Accutane). ${ }^{17}$ Isotretinoin also alters the Meibomian gland function and structure, causing acinar atrophy and hyposecretion of the Meibomian oil. All abnormal findings disappeared after one month of stopping
Correspondence: Dr. Adriana Bolekova, Department of Anatomy, Faculty of Medicine, Pavol Jozef Safarik University, Srobarova 2, 041 80 Kosice, Slovak Republic.

Tel. +421.55.234 3216.

E-mail: adriana.bolekova@upjs.sk

Key words: Meibomian glands, retinoic acid NADPH-d histochemistry, nitrergic innervation development.

Acknowledgements: this work was supported by grant VEGA 1/0154/11.

Received for publication: 31 October 2012. Accepted for publication: 17 November 2012.

This work is licensed under a Creative Commons Attribution NonCommercial 3.0 License (CC BYNC 3.0).

\section{(C) Copyright A. Bolekova et al., 2012}

Licensee PAGEPress, Italy

European Journal of Histochemistry 2012; 56:e50 doi:10.4081/ejh.2012.e50

the treatment. ${ }^{18}$ Many nerve fibers occur around and within the wall of the small vessels that build up a dense meshwork around the acini. Nitric oxide (NO) is an unconventional neurotransmitter, free radical, involved in many physiological and pathological processes. The production of NO is indicated immunohistochemically by the demonstration of nitric oxide synthase (NOS), the enzyme responsible for NO generation. The presence of constitutive NOS (neuronal and endothelial NOS) may be inferred by positive NADPH-d staining method, which is widely used in the investigation of the nervous system. This method is specific for the depiction of nerve structures and blood vessels. Staining of other structures is non-specific NADPH-d stain that is not equivalent to the presence of NOS. ${ }^{2,19}$

Previous studies have determined the localization and morphology of the NADPH-d positive neural elements of the Meibomian glands in adult rats and also during postnatal development, thereby implying participation of NO in Meibomian glands innervation. ${ }^{2,7}$ In this work the presence of NADPH-d activity in and around the Meibomian glands was investigated in order to discover the prenatal effect of excess of RA to its development.

\section{Materials and Methods}

\section{Animal treatment and tissue preparation}

The experimental animals, Wistar pups of 
both genders ( $\mathrm{n}=18)$ weighing $30-35 \mathrm{~g}$ and aged 14 days post partum were housed in cages together with their mothers under standard conditions of temperature and lighting. The animals were handled in accordance with the regulations of the Association for Research in Vision and Ophthalmology. All experiments were conducted in accordance with the Committee for Ethics on Animal Experiments at the Faculty of Medicine, Pavol Jozef Safarik University in Kosice, Slovakia, and the State Veterinary and Food Administration of the Slovak Republic No. 1757/10-221/3a approved the experimental protocol. All the experiments were made at the same hour daily and season of the year. Experimental animals were divided in two groups: control group and RA group. In the control group 16 eyelids of 14 days old rats with no experimental intervention were studied. In RA group 20 eyelids of rat pups with prenatal administration of the excess of RA were used. $1 \mathrm{mg} / \mathrm{kg}$ of all-trans RA (Sigma-Aldrich, R2625), was applied to pregnant Wistar rats of RA group intraperitoneally during the gestational period in each of the $12^{\text {th }}-14^{\text {th }}$ embryonic days, totally $3 \mathrm{mg} / \mathrm{kg}$. The upper eyelids were carefully excised after killing animals with an overdose of diethyl ether and stored in the $4 \%$ paraformaldehyde $\quad+0,1 \%$ glutaraldehyde buffered with $0,1 \mathrm{M}$ sodium phosphate, $\mathrm{pH}-7,4$ for 3-4 h. The fixatives were freshly made up immediately prior to perfusion.

\section{Histochemical procedure}

Samples were stored in the above mentioned solution for $24 \mathrm{~h}$, then $1 \mathrm{~h}$ in increasing concentrations of sucrose (15-30\%) and stored overnight at $4^{\circ} \mathrm{C}$. Both upper eyelids were sampled at their centre and sectioned parasagittaly in a freezing microtome at a thickness of $\mathbf{4 5}$ $\mu \mathrm{m}$. Ten sections/eyelid were processed with NADPH-d histochemistry using the technique according to Scherer-Singler et al., which was modified to suit our laboratory conditions, as was reported in our previous studies..$^{2,20,21}$ The sections were incubated for an hour at $37^{\circ} \mathrm{C}$ in a solution containing $1.5 \mathrm{mM}$ nitroblue tetrazolium (NBT, Sigma-Aldrich, N6876), $1.0 \mathrm{mM}$ ß-NADPH (NADPH, Sigma-Aldrich N1630), $10.0 \mathrm{mM}$ monosodium malate (malic acid, Sigma-Aldrich M1125) and 0.5\% Triton X-100 dissolved in 0,1 M phosphate buffer ( $\mathrm{pH} 7.4$ ). As a control for NADPH-d staining we carried out the processing with the omission of the substrate ß-NADPH in the reaction medium. This was to test for endogenous reduction activity in the corresponding blue formazan product. ${ }^{22}$ No residual activity was observed. Following the reaction, sections were rinsed in $0,1 \mathrm{M}$ phosphate buffer ( $\mathrm{pH} 7,4)$, mounted on glass slides, air-dried overnight and finally cover slipped with mounting medium Entellan (Merck 100869).

\section{Assessment}

The morphologic appearance of the NADPH-d positive structures in Meibomian glands were evaluated histologically, on slides using light microscope Olympus $\mathrm{CH} 20$. Images were obtained with a Moticam 2300 camera mounted on the microscope Optika B600 TI coupled to a capture board in a computer. Digitalized images were analyzed using software Motic Images Plus (version 2.0 ML).

\section{Results}

NADPH-diaphorase histochemical procedure results in the deposition of a dark blue water insoluble formazan reaction product. We observed NADPH-d positive staining in nerve fibers and endothelial cells of blood vessels coursing around the Meibomian glands, between acini and in the submucosa.
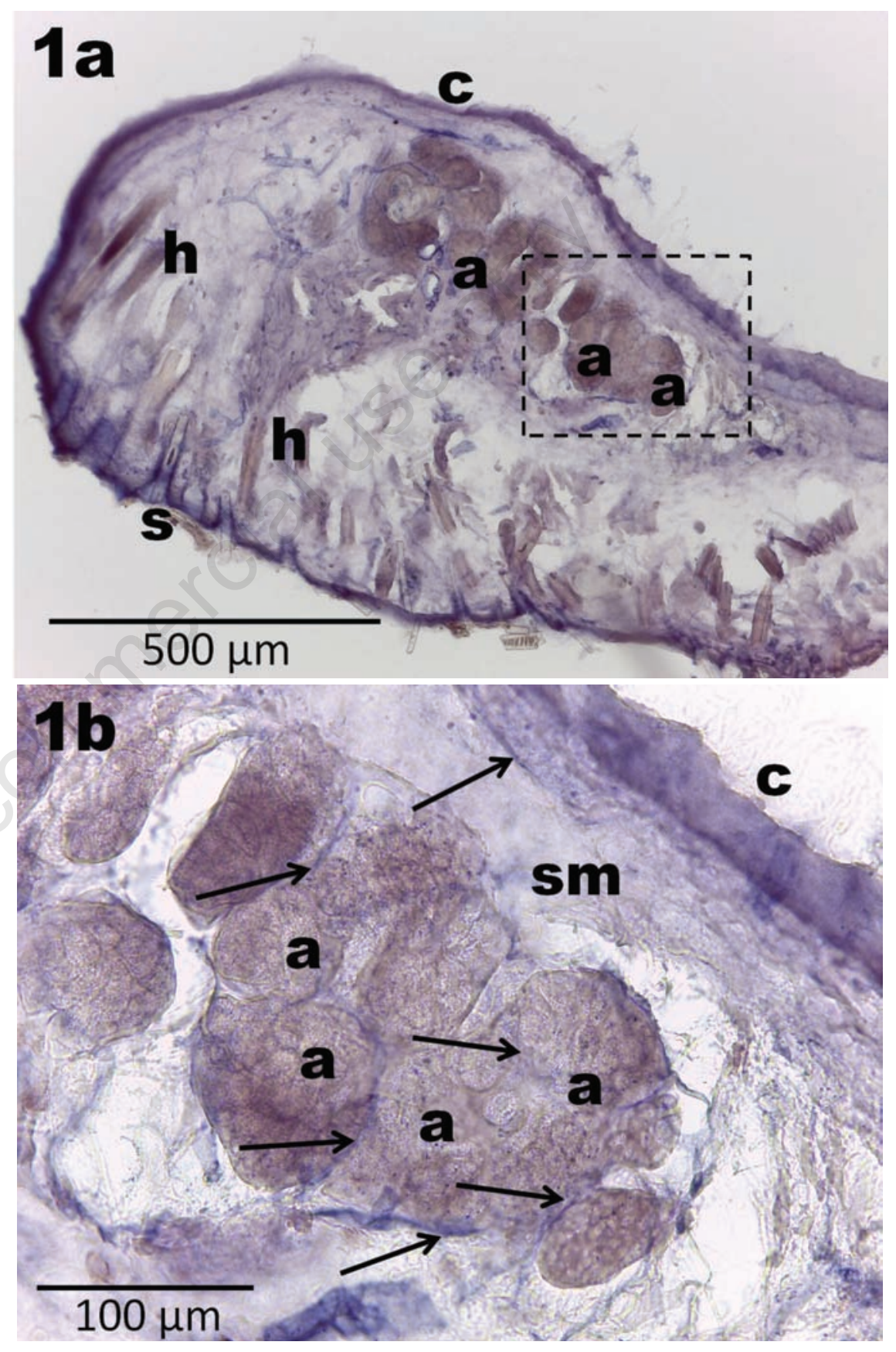

Figure 1. Parasagittal section through the rat upper eyelid on $14^{\text {th }}$ postnatal day, control group, NADPH-diaphorase staining. a) Round shaped acini of Meibomian glands (a) lie near the conjunctival surface (c) of the eyelid; skin (s); hairs (h); scale bar: $500 \mu \mathrm{m}$. b) Inset from a: blood vessels accompanied by nerve fibers (arrows) are running mostly between acini of the Meibomian glands (a) and rarely in submucosa (sm); scale bar: $100 \mu \mathrm{m}$. 
Meibomian glands were dark stained (Figures 1 and 2). Histochemical analysis of control eyelids revealed more numerous NADPH-d wellstained acini of Meibomian glands arranged tightly into groups. Darker acini were in preponderance over lightly stained acini, the majority of acini showed a uniform dark blue staining (Figure 1a). Intensively stained vessels and NADPH-d positive nerve fibers bordered acini of Meibomian glands. These structures were present in the submucosal layer as well (Figure 1b).

The analysis of RA group showed less numerous, shrunken acini of Meibomian glands, which were seen not only smaller in size, but also the density of their staining was considerably lowered (Figure 2a). In the RA group in the submucosa marked differences were noticed compared to eyelids of control group, there were more numerous NADPH-d stained vessels accompanied by NADPH-d positive nerve fibers. Although the nerve fibers and blood vessels were less numerous between the acini, only a few fine nerve fibers and blood vessels were present running between the acini of Meibomian glands (Figure 2b).

\section{Discussion}

Vitamín A and its metabolites are essential for nutrition and proper functioning of organisms. ${ }^{23-25}$ Generally accepted fact is that the excess of vitamin A during pregnancy has teratogenic effects towards offspring. ${ }^{26}$ Hence the composition of human and rat Meibomian glands are very similar, the development of nitrergic innervation in the rat's Meibomian gland was investigated in our previous experiment. ${ }^{2,27}$ NADPH-d histochemical activity in nerve fibers and vessels suggests utilization of NO. ${ }^{28}$ In this work, $14 \%$ of RA pups perished of malformations during perinatal period. Histochemical analysis revealed changes in morphology and nitrergic innervations of Meibomian glands in 14-day-old rats prenatally treated with excess of RA. Acini were smaller, shrunken and less intensively NADPH-d stained, more nerve fibers and vessels were present in submucosal layer of eyelid, although they were less between acini. These findings corresponded with results in rabbits by Lambert and Smith..$^{29}$ Mathers et al. found, that during usage of Accutane by human, Meibomian glands appeared significantly less dense and were atrophied, with consequent increased tear's evaporation and osmolarity. ${ }^{30}$ During systemic RA treatment, eye dryness was related to the dose used, known the period of treatment. ${ }^{31}$ It is very important to select such dose of RA that will have the less effect to Meibomian glands while maintaining thera- peutic effects. After a lot of pilot experiments we finally considered to use all-trans RA in doses of $1 \mathrm{mg} / \mathrm{kg}$ on pregnant rat in $14^{\text {th }}-16^{\text {th }}$ embryonic day to investigate the influence of prenatal administered subtoxic doses of RA to offspring's postnatal development. With isotretinoin usage, all glands are approximate- ly equally affected, while by influence of infectious diseases some glands are normal and others are severely affected. ${ }^{32}$ This was confirmed as well in our experiment, as all Meibomian glands were equally affected by excess of RA. Changes of Meibomian glands are reversible on discontinuing the drug,
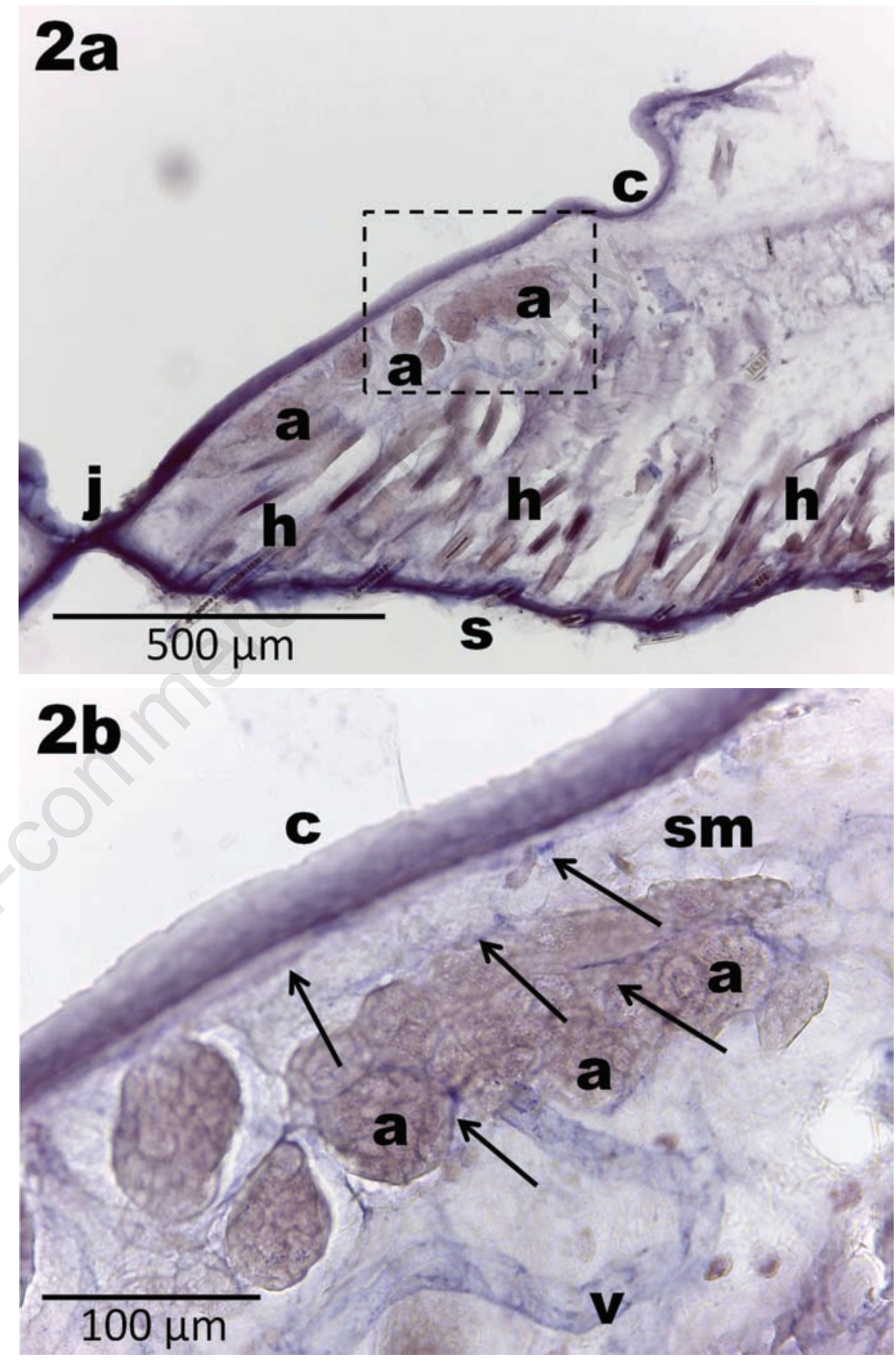

Figure 2. Parasagittal section through the rat upper eyelid on $14^{\text {th }}$ postnatal day, RA group, NADPH-diaphorase staining. a) Shrunken acini of Meibomian glands (a) lie near the conjunctival surface (c) of the eyelid; skin (s); hairs (h); junction between upper and lower eyelid (j); blood vessel (v); scale bar: $500 \mu \mathrm{m}$. b) Inset from a: blood vessels accompanied by nerve fibers (arrows) are running mostly in submucosa ( $\mathrm{sm}$ ); and rarely between acini of the Meibomian glands (a); scale bar: $100 \mu \mathrm{m}$. 
whereas Meibomian glands after prenatal treatment are altered permanently. This is the reason why, RA treatment must be forbidden during pregnancy.

The NADPH-d histochemistry was used as a method for visualisation of enzyme NOS in neurons, their fibres and blood vessel endothelia. In other tissues, positive staining for NADPH-d is non-specific. ${ }^{33,34}$ In many regions of the central and peripheral nervous system, the presence of NOS is taken to indicate a functional role for NO in the involved tissues. $^{35,36}$ This innervation appears in early development and is fully established after third postnatal week in rat nervous system. The picture of Meibomian glands of prenatally influenced rats showed developmental stagnation compared to control animals. RA may similarly cause an alteration in the composition of Meibomian gland excretions, resulting in increased viscosity. In the early phases of development, up to $1^{\text {st }}$ postnatal week it was observed that nerve fibers and blood vessels were running in the submucosa, not in the septa between the acini. In samples of adult rats, typically observed intensely stained nerve fibers running as a network around blood vessels were seen. They were mostly seen between acini and around the acinar ducts, less in the submucosal layer. Other useful investigations were also performed during rat embryonic development, and age-related changes of NADPH-diaphorase positivity in the rat were reported. ${ }^{37-40}$

The excess of retinoic acid during prenatal period influences the development and morphology of nitrergic structures of the Meibomian glands. It may be that innervation is more related to regulating the periacinar vascular network and meeting nutritional demands. Further studies are needed to explore the role of $\mathrm{NO}$ in these processes. Future functional studies of this model may yield important insights into the relationships between RA treatment and Meibomian gland morphology and innervation.

\section{References}

1. Driver PJ, Lemp MA. Major review. Meibomian gland dysfunction. Surv Ophthalmol 1996;40:343-67.

2. Kluchova D, Bolekova A, Heichel C, Bron AJ, Kozak I. NADPH-diaphorase expression in the Meibomian glands of rat palpebra in postnatal development. Eur $\mathrm{J}$ Histochem 2010;54:e47.

3. Bron AJ, Yokoi N, Gaffney E, Tiffany JM. Predicted phenotypes of dry eye: proposed consequences of its natural history. Ocul Surf 2009;7:21-35.
4. Grob S, Kozak I, Zhang K. Retinal pigment epithelial tear resembling retinal tear. Eye (Lond) 2012;26:333-4.

5. Kozak I, Freeman WR. Infectious retinitis and retinal disease in human immunodeficiency virus-infected individuals: a photographic atlas. Retina 2011;31:1-42.

6. Gudas LJ: Emerging roles for retinoids in regeneration and differentiation in normal and disease states. Biochim Biophys Acta 2012;1821:213-21.

7. Kozak I, Bron AJ, Kucharova K, Kluchova D, Marsala M, Heichel C, et al. Morphologic and volumetric studies of the Meibomian glands in elderly human eyelids. Cornea 2007;26:610-4.

8. Penniston KL, Tanumihardjo SA. The acute and chronic toxic effects of vitamin A. Am J Clin Nutr 2006;83:191-201.

9. Rhinn M, Dollé P. Retinoic acid signalling during development. Development 2012; 139:843-58.

10. Samarut E, Rochette-Egly C. Nuclear retinoic acid receptors: conductors of the retinoic acid symphony during development. Mol Cell Endocrinol 2012;348:34860.

11. Gupta R.Reproductive and developmental toxicology. Academic Press, 2011.

12. Maden M. Retinoic acid in the development, regeneration and maintenance of the nervous system. Nat Rev Neurosci 2007;8:755-65.

13. Theodosiou M, Laudet V, Schubert M. From carrot to clinic: an overview of the retinoic acid signaling pathway. Cell Mol Life Sci 2010;67:1423-45.

14. Ross SA, McCaffery PJ, Dräger UC, DeLuca LM. Retinoids in embryonal development. Physiol Rev 2000;80:1021-54.

15. Bhutani T, Koo J. A review of the chemopreventative effects of oral retinoids for internal neoplasms. J Drugs Dermatol 2011;10:1292-8.

16. Ross AC, Chen Q, Ma Y. Vitamin A and retinoic acid in the regulation of B-cell development and antibody production. Vitam Horm 2011;86:103-26.

17. McCaffery PJ, Adams J, Maden M, RosaMolinar E. Too much of a good thing: retinoic acid as an endogenous regulator of neural differentiation and exogenous teratogen. Eur J Neurosci 2003;18:457-72.

18. Bozkurt B, Irkec MT, Atakan N, Orhan M, Geyik PO. Lacrimal function and ocular complications in patients treated with systemic isotretinoin. Eur J Ophthalmol 2002;12:173-6.

19. Bolekova A, Kluchova D, Spakovska T, Toth S, Vesela J. NADPH-diaphorase expression in the rat jejunum after intestinal ischemdia/reperfusion. Eur J Histochem 2011;55: e23.
20. Scherer-Singler U, Vincent SR, Kimura H, Mc-Geer EG. Demonstration of a unique population of neurons with NADPHdiaphorase histochemistry. J Neurosci Methods 1983;9:229-34.

21. Lovasova K, Kluchova D, Bolekova A, Dorko F, Spakovska T. Distribution of NADPH-diaphorase and AChE activity in the anterior leaflet of rat mitral valve. Eur J Histochem 2010;54:e5.

22. Hope BT, Vincent SR. Histochemical characterization of neuronal NADPHdiaphorase. J Histochem Cytochem 1989; 37:653-61.

23. D'Ambrosio DN, Clugston RD, Blaner WS. Vitamin A metabolism: an update. Nutrients 2011;3:63-103.

24. Kimáková T, Bernadič M, Krištúfek P. [Antioxidants in nutrition. Issue of free radicals, oxidative stress and antioxidants in relation to non-infectious diseases, 2nd part]. [Article in Czech]. Monitor medicíny SLS 2012;1-2:9-14.

25. Rovny I, Truskova I, Bielik I, Kimakova T. [Selected chapters from public health]. [In Slovak]. Bratislava: Editio Collegium Elisabethinum, 2012.

26. Kam RK, Deng Y, Chen Y, Zhao H. Retinoic acid synthesis and functions in early embryonic development. Cell Biosci 2012; 2:e11.

27. Harvey DJ, Tiffany JM, Duerden JM, Pandher KS, Mengher LS. Identification by combined gas chromatography-mass spectrometry of constituent long-chain fatty acids and alcohols from the Meibomian glands of the rat and a comparison with human Meibomian lipids. J Chromatogr 1987;414:263.

28. Cserep C, Szonyi A, Veres JM, Nemeth B, Szabadits E, de Vente J, et al. Nitric oxide signaling modulates synaptic transmission during early postnatal development. Cereb Cortex 2011;21:2065-74.

29. Lambert RW, Smith RE. Pathogenesis of blepharoconjunctivitis complicating 13cis-retinoic acid (isotretinoin) therapy in a laboratory model. Invest Ophthalmol Vis Sci 1988;29:1559-64.

30. Mathers WD, Shields WJ, Sachdev MS, Petroll WM, Jester JV. Meibomian gland morphology and tear osmolarity: changes with Accutane therapy. Cornea 1991;10: 286-90.

31. Cumurcu T, Sezer E, Kilic R, Bulut Y. Comparison of dose-related ocular side effects during systemic isotretinoin administration. Eur J Ophthalmol 2009; 19:196-200.

32. Farrell LN, Strauss JS, Stranieri AM. The treatment of severe cystic acne with 13cis-retinoic acid. Evaluation of sebum production and the clinical response in a 
multiple dose trial. J Am Acad Dermatol 1980;3:602-11.

33. Bredt DS, Snyder SH. Nitric oxide, a novel neuronal messenger. Neuron 1992;8:3-11.

34. Oess S, Icking A, Fulton D, Govers R, Müller-Esterl W. Subcellular targeting and trafficking of nitric oxide synthases. Biochem J 2006;396:401-9.

35. Talapka P, Bodi N, Battonyai I, Fekete E, Bagyanszki M. Subcellular distribution of nitric oxide synthase isoforms in the rat duodenum. World J Gastroenterol 2011;
17:1026-9.

36. Hope BT, Michael GJ, Knigge KM, Vincent SR. Neuronal NADPH-diaphorase is a nitric oxide synthase. PNAS USA 1991;88: 2811-4.

37. Lacunza E, Ferretti V, Barbeito C, SegalEiras A, Croce MV. Immunohistochemical evidence of Mucl expression during rat embryonic development. Eur J Histochem 2010;54:e49.

38. Racekova E, Martoncikova M, Mitruskova B, Cizkova D, Orendacova J. Age related changes of NADPH-diaphorase positivity in the rat rostral migratory stream. Cell Mol Neurobiol 2005;25:1093-105.

39. Pellicciari C, Malatesta M. Identifying pathological biomarkers: histochemistry still ranks high in the omics era. Eur $\mathrm{J}$ Histochem 2011;55:e42.

40. Pellicciari C. Histochemistry through the years, browsing a long-established journal: novelties in traditional subjects. Eur J Histochem 2010;54:e51. 